\title{
Attenuation of influenza-like symptomatology and improvement of cell-mediated immunity with long-term $\mathrm{N}$-acetylcysteine treatment
}

\author{
S. De Flora*, C. Grassi**, L. Carati ${ }^{+}$
}

\begin{abstract}
Attenuation of influenza-like symptomatology and improvement of cell-mediated immunity with long-term N-acetylcysteine treatment. S. De Flora, C. Grassi, L. Carati. CERS Journal Ltd 1997.

ABSTRACT: N-acetylcysteine (NAC), an analogue and precursor of reduced glutathione, has been in clinical use for more than 30 yrs as a mucolytic drug. It has also been proposed for and/or used in the therapy and/or prevention of several respiratory diseases and of diseases involving an oxidative stress, in general. The objective of the present study was to evaluate the effect of long-term treatment with NAC on influenza and influenza-like episodes.

A total of 262 subjects of both sexes $(78 \% \geq 65 \mathrm{yrs}$, and $62 \%$ suffering from nonrespiratory chronic degenerative diseases) were enrolled in a randomized, doubleblind trial involving 20 Italian Centres. They were randomized to receive either placebo or NAC tablets $(600 \mathrm{mg})$ twice daily for 6 months. Patients suffering from chronic respiratory diseases were not eligible, to avoid possible confounding by an effect of NAC on respiratory symptoms.

NAC treatment was well tolerated and resulted in a significant decrease in the frequency of influenza-like episodes, severity, and length of time confined to bed. Both local and systemic symptoms were sharply and significantly reduced in the NAC group. Frequency of seroconversion towards $A / H_{1} N_{1}$ Singapore $6 / 86$ influenza virus was similar in the two groups, but only $25 \%$ of virus-infected subjects under NAC treatment developed a symptomatic form, versus $79 \%$ in the placebo group. Evaluation of cell-mediated immunity showed a progressive, significant shift from anergy to normoergy following NAC treatment.

Administration of $\mathrm{N}$-acetylcysteine during the winter, thus, appears to provide a significant attenuation of influenza and influenza-like episodes, especially in elderly high-risk individuals. $N$-acetylcysteine did not prevent $A / H_{1} N_{1}$ virus influenza infection but significantly reduced the incidence of clinically apparent disease.
\end{abstract}

Eur Respir J 1997; 10: 1535-1541.

*Institute of Hygiene and Preventive Medicine, University of Genoa, Italy. **Institute of Respiratory Diseases, University of Pavia, Italy. ${ }^{+}$Medical Dept, Zambon Group S.p.A., Bresso, Milan, Italy.

Correspondence: S. De Flora

Institute of Hygiene and Preventive Medicine University of Genoa

via A. Pastore 1

I-16132 Genoa

Italy

Keywords: N-acetylcysteine

cell-mediated immunity

chemoprevention

immunomodulation

influenza

influenza-like symptoms

Received: November 251996

Accepted after revision April 111997
$\mathrm{N}$-acetylcysteine (NAC) was introduced in the 1960s as a mucolytic drug, due to its ability to cleave disulphide bridges in mucous protein complexes and, thus, depolymerize mucin molecules. Subsequently, it was discovered that NAC is an effective antioxidant and enriches the intracellular sulphydryl pool, acting as a precursor of reduced glutathione (GSH), which provides the organism with crucial defence mechanisms towards toxic agents of various nature [1]. Uptake of extracellular GSH occurs largely, if not entirely, by pathways involving prior breakdown of GSH to dipeptides and amino acids, their subsequent transport into the cell, and intracellular synthesis of the tripeptide [1]. In contrast, NAC easily penetrates cell membranes and, unlike cysteine, which is the rate-limiting amino acid in GSH synthesis, has a very low toxicity [2, 3]. Therefore, the possible clinical applications of NAC cover several pathological conditions involving oxidative stress, including acute and chronic bronchitis, acute respiratory distress syndrome (ARDS), and certain cardiovascular diseases $[3,4]$.
Studies carried out in Italy [5, 6], Sweden [7, 8], the UK $[9,10]$, and Germany [11] provided evidence that prophylactic treatment with NAC reduces the incidence and duration of exacerbations in chronic bronchitis. This effect was ascribed not only to the mucolytic action of NAC and to the improved mucociliary clearance but also to an enhancement of antioxidant lung defences [3], and to modulation of virulence factors of the intrabronchial bacterial flora [12].

The generation of free radicals by phagocytes involved in the inflammatory process and alterations of the immune response play a key role in viral infections, thereby explaining the clinical manifestations resulting from the cytotoxic damage in virus-infected tissues $[13,14]$. The main symptoms in the influenza virus disease arise from the cytopathic effects in the epithelial cells lining the respiratory tract, and additionally from the release of immunoactive mediators, which trigger the cascade of events directed towards elimination of the virus [15]. At the same time, these mediators, including cytokines, such as interleukins (ILs), interferon-gamma (IFN- $\gamma$ ) and 
the tumour necrosis factor (TNF), have pleiotropic effects both locally and systemically [16]. The influenza virus disease is in fact associated with symptoms, such as asthenia and lack of appetite, which suggest an involvement of TNF, whose generation in phagocytes activated by the virus is further enhanced by the possible presence of even small traces of fragments of bacterial origin, such as lipopolysaccharides (LPS). This may explain the severe complications that frequently occur in combined influenza virus and bacterial infections [17].

These pathogenetic patterns provide the rationale for a clinical use of antioxidants in viral infections, aimed at normalizing the altered cell redox equilibrium, and at preventing and/or treating the clinical manifestations of immunological dysfunctions [18]. In this context, NAC has been assayed in experimental infection by influenza A viruses and has been shown to reduce the severity of the disease in mice $[19,20]$.

The present multicentric study, referred to as the NACIS study (acronym for $\mathrm{N}$-acetylcysteine in Immune System), was designed in order to evaluate the efficacy of NAC, administered orally throughout the cold season, in preventing the occurrence and reducing the severity of influenza-like episodes in general, and specifically those caused by the influenza $\mathrm{A} / \mathrm{H}_{1} \mathrm{~N}_{1}$ virus. Another goal of this study was to assess the effect of NAC treatment on cell-mediated immunity.

\section{Materials and methods}

\section{Design of the study}

A multicentre study was designed according to a controlled, randomized and double-blind model. The study was authorized by the Italian Ministry of Health (Authorization No. 800.C.35/75.828 dated October 1, 1991) and was approved by the Ethics Committees of all participating institutions. The study complied with the declaration of Helsinki, in the Hong Kong (1989) revision. Informed consent was received from all subjects prior to randomization in one of the two treatment groups.

The 20 participating Centres were distributed over the whole country, in order to cover possible variations related to geographically uneven socioeconomic, climatic and epidemiological situations.

\section{Subjects}

A total of 262 individuals of both sexes were recruited (table 1). Patients suffering from chronic respiratory diseases were not eligible, in order to avoid possible confounding by an effect of NAC on respiratory symptoms. The criteria for eligibility were: the presence of chronic degenerative diseases (other than those of the respiratory tract) in subjects aged $\leq 64$ yrs; or aged $\geq 65$ yrs (irrespective of the presence of chronic nonrespiratory diseases). Overall, people of $\geq 65$ yrs accounted for $78 \%$ of subjects recruited. Subjects having been vaccinated against influenza in the same season or in the previous season were not eligible. As shown in table 1, sex and age, as well as anthropometric, lifestyle and clini-
Table 1. - Characteristics of the subjects under study

\begin{tabular}{lcc}
\hline & \multicolumn{2}{c}{ Treatment } \\
Characteristics & Placebo & NAC \\
\hline Recruited subjects n & 129 & 133 \\
Sex M/F n & $48 / 81$ & $60 / 73$ \\
M/F \% & $37 / 63$ & $45 / 55$ \\
Age yrs & $68 \pm 11$ & $69 \pm 11$ \\
& $(33-92)$ & $(23-92)$ \\
Age $\geq 65$ yrs n & $99(77)$ & $105(79)$ \\
$\leq 64$ yrs n & $30(23)$ & $28(21)$ \\
Weight kg & $67 \pm 11$ & $71 \pm 13$ \\
Height cm & $163 \pm 9$ & $165 \pm 9$ \\
Smoking habits n & $19(15)$ & $11(8)$ \\
Smokers & $18(14)$ & $23(17)$ \\
Ex-smokers & $57(44)$ & $65(49)$ \\
Nonsmokers & $35(27)$ & $34(26)$ \\
Unknown & $110(85)$ & $112(84)$ \\
Residence area n & $19(15)$ & $21(16)$ \\
Urban/industrial & $39(30)$ & $45(34)$ \\
Rural & $15(12)$ & $20(15)$ \\
Chronic disease & $11(9)$ & $12(9)$ \\
Cardiovascular & $9(7)$ & $5(4)$ \\
Osteoarticular & $3(2)$ & $3(2)$ \\
Metabolic & $52(40)$ & $48(36)$ \\
CNS and psychosomatic & & \\
Miscellaneous & $59(46)$ & $52(39)$ \\
None & $16(12)$ & $14(11)$ \\
Previous influenza-like & & \\
episodes (last 2 yrs) n & & \\
Total & & \\
Complicated & & \\
\hline
\end{tabular}

Data are presented as mean \pm SD and range in parenthesis, or as absolute value and percentage in parenthesis. NAC: N-acetylcysteine; M: male; F: female; CNS central nervous system.

cal characteristics of the subjects randomized in the placebo group and in the NAC group were well matched, without any statistically significant difference between the two treatment groups.

\section{Treatments}

Effervescent tablets of NAC (Fluimucil; 600 mg.tablet $^{-1}$ ) or of its excipient (placebo), whose appearance was indistinguishable from that of the drug, were prepared by Zambon Group (Bresso, Milan, Italy). Each batch, containing the 360 tablets sufficient to treat each individual for a period of 6 months (two tablets per day, after breakfast and at bedtime), was labelled and coded with a sequential number, corresponding to the randomization created by the computer. Randomization by blocks of four subjects was made within each Centre. Each Centre was provided with drug samples sufficient to treat a number of subjects, ranging 12-16. The overall requirement of drug was divided into 6 monthly batches, which were assigned to each subject at the time of his/her first enrolment and at subsequent medical examinations. In order to check compliance with treatment, the subjects were instructed to hand back unused tablets at each examination. The NAC dosage was chosen based on the extensive clinical experience showing the high tolerability of this drug, even at much higher doses than those commonly prescribed as a mucolytic agent $[2,3]$. 
Treatment started either in October or November 1991, and was planned to continue for 6 months, i.e. until April or May 1992, respectively. The use of drugs for symptom relief, such as antipyretics, was left to the discretion of the attending physician, who registered all such treatments in the case report forms. In the meantime, those subjects who suffered from chronic degenerative diseases were allowed to continue the treatments for controlling their basal pathological condition. Use of immunomodulants, vitamins or antioxidants was, however, prohibited.

\section{Clinical evaluations}

Each subject was supplied with a personal diary card, and instructed to fill in any sign and/or symptom suggestive of an influenza-like episode, based on a suitable check list. Influenza-like episodes were assessed based on the presence of two or more of the following signs and/or symptoms: fever $\left(\geq 38^{\circ} \mathrm{C}\right)$, asthenia, lack of appetite, headache, myalgia/arthralgia, coryza/rhynorrhoea, sore throat, catarrh and cough. At monthly intervals, the clinicians evaluated the diary card and performed a complete medical examination. The patients were also asked to contact the clinicians soon after the onset of any sign or symptom, and, whenever necessary, additional clinical examinations were performed.

A severity score was constructed for each episode, on the basis of the recorded data and of clinical evaluation, by ascribing score 1 to each sign or symptom, except for fever $\left(\geq 38^{\circ} \mathrm{C}\right)$, to which score 2 was ascribed. Episodes with total scores of 2-3, 4-5 and $>5$ were classified as mild, moderate and severe, respectively. Moreover, information was available on the duration of each episode, length of time in bed, and symptomatic therapy, when needed. In order to evaluate tolerability to treatments, any side-effect occurring during the observation period was carefully recorded.

Additionally, blood samples were collected at the start and at the end of the 6 month follow-up period, in order to assess routine haematological and biochemical parameters.

\section{Immunological evaluations}

Antibodies towards the $\mathrm{A} / \mathrm{H}_{1} \mathrm{~N}_{1}$ Singapore 6/86 influenza virus were measured by haemagglutination inhibition in basal and final blood serum samples from all subjects evaluable in the study, using a standard procedure [21]. An increase in antibody titre of $\geq$ fourfold in the final serum sample, compared to the basal sample, was considered to be indicative of a specific seroconversion.

Cell-mediated, delayed sensitivity was evaluated in all subjects at the time of enrolment and after 1, 3 and 6 months by using a skin applicator in polyacrylic resin, loaded with seven antigens (tetanus, diphtheria, Streptococcus group C, tuberculin, Proteus mirabilis, Tricophyton mentagrophytes and Candida albicans) and their carrier (glycerol) (Multitest IMC, Istituto Merieux Italia, Rome, Italy). The results were read $48 \mathrm{~h}$ after the application and expressed in terms of "average composite score", which takes into account the number of positive anti- gens and the diameter of the skin reaction. In particular, the subjects were classified as: anergic in case of total absence of skin reactivity to any antigen; hypoergic in case of skin reactivity to $\leq 2$ antigens yielding a cumulative skin reaction diameter $<10 \mathrm{~mm}$; and normoergic in case of skin reactivity either to $\geq 3$ antigens or $\geq 2$ antigens when the cumulative skin reaction diameter was $\geq 10 \mathrm{~mm}$.

\section{Statistical analysis}

Comparisons of data in the placebo group and in the NAC group were made by Student's t-test in case of continuous variables (e.g. age, duration of treatment, etc.), after having checked for normality of distribution, and by Chi-squared $\left(\chi^{2}\right)$ analysis in case of nominal variables expressed as frequencies. All statistical procedures were run in a Macintosh Quadra 840/AV PC using the SPSS International program. A p-value of less than 0.05 was considered significant.

\section{Results}

\section{Compliance and tolerability}

Compliance to treatment was quite satisfactory, since 93\% of subjects receiving the placebo and $94 \%$ of NACtreated subjects took more than $80 \%$ of the allotted drug (table 2).

The treatment with $600 \mathrm{mg}$ NAC twice daily was well tolerated in the large majority of subjects, with only $9 \%$

Table 2. - Compliance with treatment, adverse effects, and frequency of influenza-like episodes as related to treatments

\begin{tabular}{lcc}
\hline & \multicolumn{2}{c}{ Treatment } \\
Variable & Placebo & NAC \\
\hline Compliance with treatment* $\mathrm{n}$ & $120(93)$ & $125(94)$ \\
Adverse events $\mathrm{n}$ & $7(5)$ & $12(9)$ \\
$\quad$ Total & 0 & $2(2)$ \\
Dysuria & $3(2)$ & $4(3)$ \\
Epigastralgia & $1(1)$ & $1(1)$ \\
Nausea/vomiting & 0 & $1(1)$ \\
Constipation & $3(2)$ & $2(2)$ \\
Diarrhoea & 0 & $2(2)$ \\
Flushing & $1(1)$ & $1(1)$ \\
Nonevaluable patients n & $6(5)$ & $6(5)$ \\
Adverse events before first control & $122(95)$ & $126(95)$ \\
Lost to follow-up & $167 \pm 34$ & $166 \pm 35$ \\
Patients evaluable for efficacy $\mathrm{n}$ & $(32-199)$ & $(33-206)$ \\
Duration of treatment days & $116(90)$ & $108(81)$ \\
& & \\
Treatment $\geq 5$ months $\mathrm{n}$ & $62(51)$ & $37(29) \dagger$ \\
Influenza-like symptomatic cases $\mathrm{n}$ & $36(30)$ & $24(19)$ \\
Total cases & $18(15)$ & $11(9)$ \\
Patients suffering from 1 episode & $2(7)$ \\
Patients suffering from 2-3 episodes & $2(2)$ \\
Patients suffering from $\geq 4$ episodes & $8(7)$ & \\
\hline
\end{tabular}

Data are presented as mean \pm SD and range in parenthesis, or as absolute value and percentage in parenthesis. *: drug or placebo intake $\geq 80 \%$. NAC: N-acetylcysteine. ": significantly lower than in the placebo group $(\mathrm{p}=0.0006)$, as assessed by Chi-squared analysis. 
reporting adverse events, a figure which was not significantly different from that of the placebo group (5\%). None of the individual adverse events reported in table 2 was significantly different in the two groups, and only $3 \%$ (NAC) and 2\% (placebo) of subjects dropped out due to the occurrence of adverse events. All laboratory end-points monitored and main vital parameters, such as cardiac frequency and arterial pressure, were unchanged at the end of the study compared to the start. In the placebo and the NAC group 19\% and 14\%, respectively, were lost to follow-up before the end of the study, but only $5 \%$ in each group could not be evaluated due to early drop out, before the first control. Thus, overall, only 14 of the recruited subjects (seven per group) could not be evaluated for the efficacy of treatment (table 2). The average observation time for the evaluable subjects was 166 days in both groups, 224 subjects (90\%) remaining under clinical observation for 5 months, and 192 subjects $(77 \%)$ for 6 months, i.e. until the expected end of the study.

\section{Frequency of influenza-like clinical cases}

Table 2 reports the frequencies of cases displaying an influenza-like symptomatology, according to the criteria indicated in "Materials and methods". The overall frequency of patients suffering from influenza-like episodes out of those evaluable for efficacy was significantly lower $(\mathrm{p}=0.0006)$ in NAC-treated subjects $(29 \%)$ than in placebo-treated subjects $(51 \%)$. It is noteworthy that, at the beginning of the study, none of the subjects recruited was suffering from respiratory symptoms.

When analysing the influenza-like episodes on a monthly basis (fig. 1), the differences between NAC-treated and placebo-treated subjects became sharp and statistically significant during the seasonal period of highest incidence of this type of disease, i.e. after 2 months (12 versus $21 \%$; $\mathrm{p}=0.046), 3$ months $(13$ versus $30 \%$; $\mathrm{p}=0.001)$, and 4 months (6 versus $16 \%$; $\mathrm{p}=0.006)$. The difference was still evident but not statistically significant after 5 months (5 versus 9\%) and 6 months (2 versus 5\%).

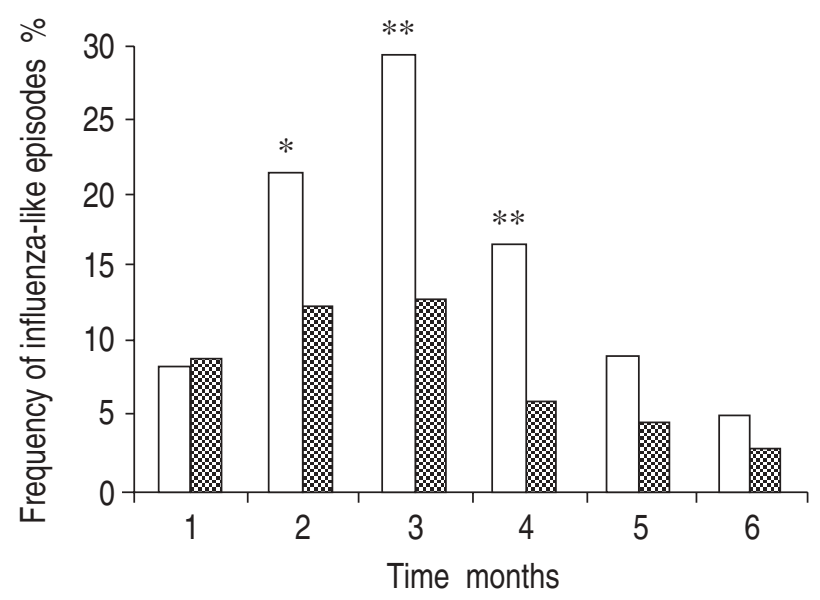

Fig. 1. - Effect of N-acetylcysteine (NAC) treatment on the frequency of influenza-like episodes during the 6 months of treatment.

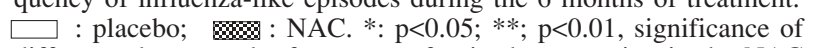
difference between the frequency of episodes occurring in the NAC group and in the placebo group, as assessed by Chi-squared analysis.

\section{Severity of influenza-like clinical cases}

Of the 99 influenza-like episodes occurring in 62 subjects within the placebo group, $47(48 \%)$ were classified as mild, $46(47 \%)$ as moderate, and $6(6 \%)$ as severe, according to the criteria reported in "Materials and methods". Of the 46 influenza-like episodes occurring in 37 subjects within the NAC group, $33(72 \%)$ were classified as mild, $12(26 \%)$ as moderate, and $1(2 \%)$ as severe. As assessed by $\chi^{2}$ analysis, the frequency of mild forms was higher in the NAC group than in the placebo group, with a difference approaching the significance threshold $(\mathrm{p}=0.06)$, whereas the frequency of moderate forms was significantly higher in the placebo group than in the NAC group $(\mathrm{p}=0.02)$. The differences were particularly pronounced by mid-season, after 3 months of treatment, when mild, moderate and severe episodes were 9, 22 and 3 in the placebo group and 8, 4 and 0 in the NAC group, respectively ( $\mathrm{p}=0.002)$, and after 4 months, when mild, moderate and severe episodes were 10, 6 and 0 in the placebo group and 3,0 and 0 in the NAC group, respectively $(\mathrm{p}=0.006)$.

When computing the number of episodes with more specific influenza-like symptoms (fig. 2), it was evident that treatment with NAC had a marked and significant protective effect both towards local symptoms, such as coryza, rhinorrhoea, sore throat, catarrh and cough, and general symptoms, especially headache and myalgiaarthralgia.

An additional criterion for evaluating the severity of influenza-like episodes was the length of time in bed which, irrespective of the age of patients, was remarkably shorter in NAC-treated subjects. In fact, in the 10 subjects suffering from influenza-like episodes who were

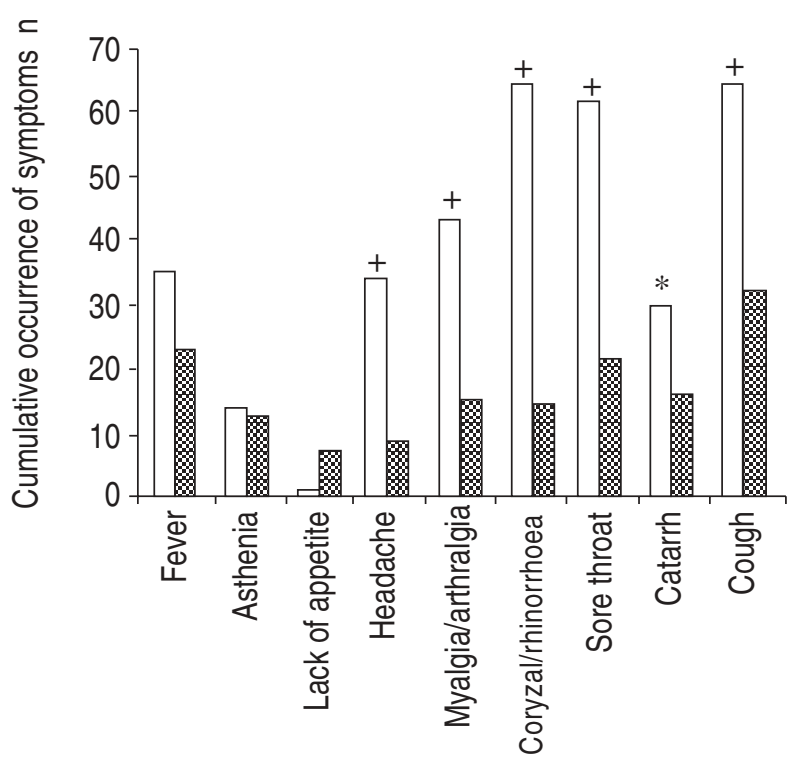

Fig. 2. - Effect of N-acetylcysteine (NAC) treatment on the cumulative occurrence of individual influenza-like signs and symptoms throughout the duration of the study. $\square$ : placebo; $\%$ : NAC. *: $\mathrm{p}<0.05$; $^{+}$: $\mathrm{p}<0.0001$, significance of difference between the frequency of symptoms in the NAC group and the placebo group, as assessed by Chi-squared analysis. Note that the data reported in this figure also include isolated signs and/or symptoms whereas, as indicated in "Materials and methods", episodes are assessed based on the presence of at least two signs and/or symptoms. 
not bedridden, nine were under NAC treatment. The proportion of patients on NAC treatment was 10 out of 12 patients bedridden for 1 day, 10 out of 20 bedridden for 2 days, eight out of 25 bedridden for 3 days, six out of 24 bedridden for 4-5 days, and only three out of 25 bedridden for more than 6 days.

The mean duration of time in bed confirmed that the severity of the disease was consistently and remarkably lower in NAC-treated subjects, with statistically significant differences after 2 months (mean \pm SD: $1.8 \pm 2.0$ days in the NAC group versus $3.2 \pm 1.0$ days in the placebo group; $\mathrm{p}<0.05$, as assessed by Student's t-test), 3 months (1.3 \pm 1.7 versus $3.2 \pm 1.4 ; \mathrm{p}<0.01), 4$ months $(0.9 \pm 1.2$ versus $2.5 \pm 1.1 ; \mathrm{p}<0.001)$, and 5 months $(0.7 \pm 0.8$ versus $1.8 \pm 0.8 ; \mathrm{p}<0.01)$. In more than half $(55 \%)$ of the episodes recorded in both groups no symptomatic therapy was applied. In the other cases, antipyretics such as aspirin and paracetamol were administered after recording the symptoms, with an almost identical frequency in the two groups. Antibiotics were used, either alone or in association with antipyretics, in 21 and $15 \%$ of the patients treated with placebo and NAC, respectively, and mainly in elderly subjects at a higher risk for bacterial complications. In no case was hospitalization needed, and no death was recorded in either group throughout the duration of the study.

\section{Frequency of influenza infection and disease}

Of the 248 subjects evaluable for assessing the efficacy of the treatments, $65(26 \%)$ underwent seroconversion towards the $\mathrm{A} / \mathrm{H}_{1} \mathrm{~N}_{1}$ Singapore $6 / 86$ virus. The frequencies of seroconversion were similar in the placebo group (24\%) and in the NAC group (29\%). However, as shown in table 3 , there was a striking protective effect with respect to the clinically apparent disease. In fact, only $25 \%$ of the virus-infected subjects under NAC treatment developed a symptomatic form, versus the $79 \%$ in the placebo group $(\mathrm{p}<0.0001)$. On the other hand, among the subjects who failed to undergo seroconversion towards the same virus, the proportion of symptomatic cases was lower in the NAC group than in the placebo group, but not to a significant extent.

\section{Effect of NAC treatment on cell-mediated immunity}

Figure 3 reports the results concerning cell-mediated immunity, which was evaluated at the beginning of the

Table 3. - Distribution of subjects as related to seroconversion towards $A / H_{1} N_{1}$ Singapore 6/86 influenza virus and to the presence or absence of symptomatology

\begin{tabular}{lccccc}
\hline \multirow{2}{*}{ Subjects } & \multicolumn{4}{c}{ Treatment } \\
& \multicolumn{2}{c}{ Placebo } & & \multicolumn{2}{c}{ NAC } \\
\cline { 2 - 3 } \cline { 6 - 7 } & $\mathrm{n}$ & $\%$ & $\mathrm{n}$ & $\%$ \\
\hline Seroconversion & $29 / 122$ & $(24)$ & & $36 / 126$ & $(29)$ \\
$\quad$ Symptomatic episodes & $23 / 29$ & $(79)$ & & $9 / 36$ & $(25)^{+}$ \\
$\quad$ No symptomatic episodes & $6 / 29$ & $(21)$ & & $27 / 36$ & $(75)^{+}$ \\
No seroconversion & $93 / 122$ & $(76)$ & & $90 / 126$ & $(71)$ \\
Symptomatic episodes & $39 / 93$ & $(42)$ & & $28 / 90$ & $(31)$ \\
No symptomatic episodes & $54 / 93$ & $(58)$ & $62 / 90$ & $(69)$ \\
\hline
\end{tabular}

NAC: $\mathrm{N}$-acetylcysteine. ${ }^{+}$: significantly different from the placebo group $(\mathrm{p}<0.0001)$, as assessed by Chi-squared analysis.

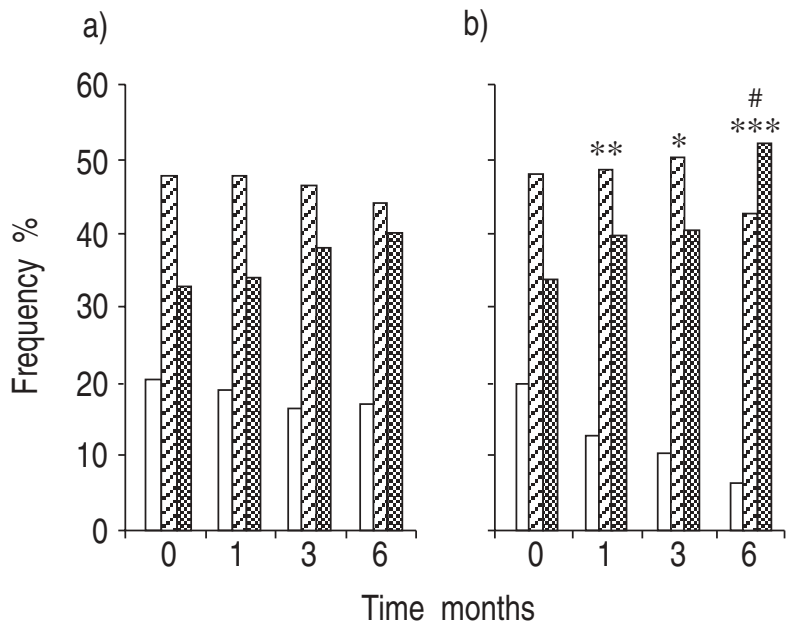

Fig. 3. - Effect of N-acetylcysteine (NAC) treatment on cell-mediated immunity. a) Placebo group; b) NAC-treated group. $\square$ : anergy; 22 : hypoergy; $\approx$ : normoergy. $*$ : $<<0.05$; $* *:$ p $<0.01$; $* * *: p<0.001$, significance of difference in the frequency of anergy, within the NAC group, after 1,3 and 6 months, compared to the start of the study (time 0); $\#: p<0.05$, significance of difference in the frequency of anergy between the NAC group and the placebo group, as assessed by Chi-squared analysis.

study (November or December, 262 subjects), one month later (December or January, 248 subjects), 3 months later (February or March, 235 subjects), and at the end of the study (April or May, 210 subjects). At the beginning of the study, the distribution of subjects according to cell-mediated reactivity was quite similar in the placebo group and in the NAC group. Thereafter, a timerelated shift of anergic condition to normoergic condition was observed in the NAC group, with variations that were statistically significant throughout the period of observation, as compared to the basal situation. In contrast, there was no significant variation in the placebo group. At the end of the study, the frequency of subjects with anergy was significantly lower in the NAC group than in the placebo group.

\section{Discussion}

The results of the NACIS multicentric study confirmed the safety and tolerability of the oral administration of NAC, even at rather high daily doses $(1,200$ $\left.\mathrm{mg} \cdot \mathrm{day}^{-1}\right)$ and for six consecutive months covering the cold season. Evidence was provided for the first time that, compared to subjects receiving a placebo, the occurrence of influenza-like episodes was significantly decreased by NAC treatment in a population sample which was mainly composed of elderly subjects and patients suffering from chronic, nonrespiratory diseases. Prevention by NAC of influenza-like symptomatic episodes was particularly evident during the winter season (December 1991 to March 1992), i.e. the period of maximum spread of viral respiratory diseases, including influenza in Europe [22]. In addition, the severity of episodes was significantly attenuated in NAC-treated subjects, as inferred from the scores adopted for evaluating the clinical severity as well as from the mean length of time in bed, which was sharply shortened in patients treated with NAC. 
The frequency of seroconversion towards the $\mathrm{A} / \mathrm{H}_{1} \mathrm{~N}_{1}$ influenza virus, which had not appreciably circulated in Italy during the previous two seasons [23, 24], was not affected by treatment, which indicates that administration of NAC failed to prevent subclinical infections. However, NAC prevented the symptomatic forms caused by $\mathrm{A} / \mathrm{H}_{1} \mathrm{~N}_{1}$ influenza virus quite efficiently, since the large majority of infected subjects in the placebo group (79\%) developed clinically apparent disease, versus only $25 \%$ in the NAC group.

It is noteworthy that NAC was successful in significantly preventing most symptoms related to influenzalike episodes, including local symptoms in the respiratory tract, such as coryza, rhinorrhoea, sore throat, catarrh and cough, and general symptoms, such as headache and myalgia-arthralgia. These patterns suggest that the protective effects of this thiol do not merely depend on its mucolytic action but are likely to be additionally ascribed to other mechanisms, such as antioxidant and immunomodulating mechanisms. The hypothesis had already been advanced 30 yrs ago that NAC protected experimental mice from influenza $A$ virus by unknown mechanisms other than the physical removal of virus particles following clearance of liquefied mucus [19]. In the same animal model, the levels of xanthine oxidase in serum and lung, and the levels of xanthine oxidase, IL- 6 and TNF in bronchoalveolar fluid were considerably enhanced by influenza A virus infection [20].

The attenuation of influenza-like symptoms in subjects under NAC treatment was accompanied by modulation of the cell-mediated immunity. At the start of the study, there was a rather high prevalence of anergic and hypoergic subjects, which reflects the age composition of the population under study [25]. Treatment with NAC produced a progressive shift from anergy to normoergy in a significant number of subjects, whereas no such effect was observed in the placebo group. These findings appear to confirm, in a clinical study, the experimental evidence concerning the immunomodulant properties of NAC [26, 27]. In addition, NAC is known to play a regulatory role on the immune system by modulating production and release of cytokines [18], which is crucial in the case of TNF, whose excessive production contributes to the cytotoxic effects occurring in virus-infected tissues as well as at a systemic level [16, 17]. These mechanisms are particularly important in elderly subjects and/or in patients affected by chronic pathological conditions, as was the case for the subjects investigated in the present study. The above categories of subjects are well-known to be particularly vulnerable in case of influenza and other viral respiratory diseases, due to a general impairment of defences, including a loss of antioxidants and a reduction of immunocompetent system functionality $[25,28]$.

In conclusion, long-term treatment with oral $\mathrm{N}$-acetylcysteine during the cold season appears to significantly attenuate the frequency and severity of influenza and influenza-like clinical episodes in elderly subjects and/or patients suffering from chronic nonrespiratory diseases. The absence of chronic respiratory diseases was chosen as a criterion of eligibility in order to avoid biases, which may have resulted from the basal disease as well as from the mucolytic activity of this drug. $\mathrm{N}$-acetylcysteine can be expected to be even more beneficial in patients suf- fering from chronic obstructive pulmonary diseases, in whom this thiol has already been shown to reduce frequency and severity of exacerbations [5-11]. Our study shows that the use of a highly tolerable drug such as $\mathrm{N}$-acetylcysteine during the cold season is especially advisable in elderly people and high-risk individuals. This may be combined with the vaccine formulated each year to include the most recent isolates of influenza viruses. At variance with the vaccine, the pharmacological prophylaxis is not virus-specific and may provide a broad-spectrum protection.

Acknowledgements: The authors are indebted to all clinicians and researchers contributing to the NACIS study. They thank Zambon Group (Milan, Italy) for supplying the pharmaceutical preparations and all materials needed for the study.

\section{Appendix}

This paper was written on behalf of the N-acetylcysteine in Immune System (NACIS) Group. The role of participating investigators and Italian institutions are listed below.

Recruitment, follow-up and clinical evaluation of patients, and evaluation of cell-mediated immunity: O. Anzano, A. Goglia (Monaldi Hospital, Naples); F. Cacciapuoti (University of Naples); A. Cipri (C. Forlanini Hospital, Rome); P. Collini (University of Pavia); S. Corallo, P. Berardinelli (Pio Albergo Trivulzio, Milan); G. Lauriello, A. Berra (S. Giovanni da Procida Hospital, Salerno); E. Paciaroni, P. Bolognini (Geriatric Hospital, Ancona); G. Palmieri (co-ordinator for northern Italy), L. Bucci (Ca' Granda Hospital, Niguarda, Milan); A. Pezza (co-ordinator for central Italy), F. De Blasio (University of Naples); S. Piantadosi, C. Infante (S. Angelo dei Lombardi Civil Hospital, Avellino); R. Rimoldi (Ospedale di Circolo, Varese); E. Rolandi, A. Garibaldi (University of Genoa); G. Salvioli, L. Serafini, C. Spinozzi (Estense Hospital, Modena); S. Scoccia, G. Schiraldi (Ca' Granda Hospital, Niguarda, Milan); S. Scoditi, M. Bisconti (Galateo Hospital, Lecce); T. Todisco (co-ordinator for central Italy), M. Dottorini (Silvestrini Hospital, S. Andrea delle Fratte, Perugia); F. Triscari (Desio Hospital, Milan).

Chemico-clinical analyses: F. Bistoni, A. Vecchiarelli (University of Perugia); P. Ferrante (Don C. Gnocchi Foundation IRCCS, Milan); L. Romano (Higher School of Immunology "Ruggero Ceppellini", Naples).

Evaluation of anti-influenza antibody: P. Ferrante (Don C. Gnocchi Foundation IRCCS, Milan).

\section{References}

1. Meister A. Metabolism and function of glutathione. In: Dolphin D, Poulson R, Avramovich O, eds. Glutathione: Chemical, Biochemical and Medical Aspects. New York, John Wiley, 1989; pp. 367-374.

2. De Flora S, Balansky R, Bennicelli C, et al. Mechanisms of anticarcinogenesis: the example of $\mathrm{N}$-acetylcysteine. In: Ioannides C, Lewis DFV, eds. Drugs, Diet and Disease., Vol. 1. Mechanistic Approaches to Cancer. Hemel Hempstead, UK, Horwood Ellis, 1995; pp. 151-203.

3. Crystal RG, Bast A, eds. Oxidants and antioxidants: 
pathophysiologic determinants and therapeutic agents. Am J Med 1991; 91: 1-145.

4. Flanagan RJ, Meredith TJ. Use of N-acetylcysteine in clinical toxicology. Am J Med 1991; 91: 131-139.

5. Grassi C, Morandini GC. A controlled trial of intermittent oral acetylcysteine in the long-term treatment of chronic bronchitis. Eur J Clin Pharmacol 1976; 9: 393-396.

6. Multicenter Study Group. Long-term acetylcysteine in chronic bronchitis: a double-blind, controlled study. Eur J Respir Dis 1980; 61: 93-108.

7. Boman G, Backer U, Larsson S, Melander B, Wahlander L. Oral acetylcysteine reduces exacerbation rate in chronic bronchitis: report of a trial organized by the Swedish Society for Pulmonary Diseases. Eur J Respir Dis 1983; 64: 404-415.

8. Rasmussen JB, Glennow G. Reduction in days of illness after long-term treatment with $\mathrm{N}$-acetylcysteine controlled release tablets in patients with chronic bronchitis. Eur J Respir Dis 1988; 1: 351-355.

9. British Thoracic Society Research Committee. Oral Nacetylcysteine and exacerbation rate in patients with chronic bronchitis and severe airways obstruction. Thorax 1985; 40: 832-835.

10. Parr DG, Huitson A. Oral N-acetylcysteine in chronic bronchitis. Br J Dis Chest 1987; 81: 341-348.

11. Meister R. Long-term therapy with $\mathrm{N}$-acetylcysteine controlled release tablets in patients with chronic bronchitis: a double-blind, placebo-controlled study (in German). Forum Prakt Allg Arzt 1986; 25: 18-22.

12. Riise GC, Larsson S, Larsson P, Jeanson S, Andersson BA. The intrabronchial microbial flora in chronic bronchitis patients: a target for $\mathrm{N}$-acetylcysteine therapy? Eur Respir J 1994; 7: 94-101.

13. Rouse BT, Horohov DW. Immunosuppression in viral infections. Rev Infect Dis 1986; 8: 850-873.

14. Maeda H, Akaike T. Oxygen-free radicals as pathogenic molecules in viral diseases. Proc Soc Exp Biol Med 1991; 198: 721-727.

15. Ada GL, Jones PD. The immune response to influenza infection. Curr Top Microbiol Immunol 1986; 128: 1-54.

16. Hennet T, Ziltener HJ, Frei K, Peterhans E. A kinetic study of immune mediators in the lungs of mice infected with influenza virus. J Immunol 1992; 149: 932-939.
17. Nain M, Hinder F, Gong JH, et al. Tumor necrosis factor production of influenza A virus-infected macrophages and potentiating effect of lipopolysaccharides. J Immunol 1990; 145: 1921-1928.

18. Peristeris P, Clark BD, Gatti S, et al. N-acetylcysteine and glutathione as inhibitors of tumor necrosis factor production. Cell Immunol 1992; 140: 390-399.

19. Streightoff F, Redmann CE, Delong DC. In vivo antiviral chemotherapy: anti-influenza action of compounds affecting mucous secretions. Antimicrob Agents Chemother 1967; 503-508.

20. Ungheri D, Pinasi C, Sanson G, et al. N-acetylcysteine activity in influenza virus infection: pathogenetic role of xanthine oxidase and tumor necrosis factor (Abstract in Italian). In: Proc. XII Congresso Società Italiana di Immunologia e Immunopatologia, Giardini Naxos, Messina, Italy, 7-10 October 1992, p. 331.

21. Lennette EH, Schmidt NJ, eds. Diagnostic procedures for viral, rickettsial and chlamydial infections. Washington DC, American Public Health Association, 1979.

22. Anonymous. Influenza in the world, 1 October 1991-30 September 1992. Weekly Epidemiol Rec (WHO, Geneva, Switzerland) 1992; 67: 373-379.

23. Anonymous. Influenza in the world, 1 October 1989-30 September 1990. Weekly Epidemiol Rec (WHO, Geneva, Switzerland) 1990; 65: 353-358.

24. Anonymous. Influenza in the world, 1 October 1990-30 September 1991. Weekly Epidemiol Rec (WHO, Geneva, Switzerland) 1991; 66: 369-374.

25. Verhaegen H, De Cock W, De Cree J, Goldstain G. Restoration of the impaired lymphocyte stimulation in old people by thymopoietin pentapeptide. J Clin Lab Immunol 1981; 6: 103-105.

26. Ohman L, Dahlgren C, Follin P, Lew D, Stendahl O. Nacetylcysteine enhances receptor-mediated phagocytosis by human neutrophils. Agents Action 1992; 36: 271-277.

27. Oddera S, Silvestri M, Sacco O, Eftimiadi C, Rossi GA. $\mathrm{N}$-acetylcysteine enhances in vitro the intracellular killing of Staphylococcus aureus by human alveolar macrophages and blood polymorphonuclear leukocytes and partially protects phagocytes from self-killing. J Lab Clin Med 1994; 124: 293-301.

28. Weksler ME. Age-associated changes in the immune response. J Am Geriatr Soc 1983; 30: 718-723. 